\title{
Trapped mountain waves with critical level just below the surface
}

\author{
C. Soufflet, F. Lott, and F. Damiens \\ Laboratoire de Météorologie Dynamique, PSL Research University, Ecole \\ Normale Supérieure, Paris
}

Received* 17 October 2018; Accepted: 14 February 2019

Keywords: Mountain waves, Trapped waves, Kelvin-Helmholtz, Instabilities, Critical level

\begin{abstract}
The trapped mountain waves produced when the incident wind near the surface is small compared to its value aloft are analyzed with a theory adapted from [Long(1953)] and compared to fully nonlinear simulations done with WRF. Although small near surface incident winds naturally occur in fronts via combination of the thermal wind balance and of the boundary layer, they pose at least two problems in mountain meteorology: zero surface incident winds produce no wave in the fully linear case, they also correspond to places where mountain waves have a critical level.

Despite these problems, the theory and WRF show that for small mountains (i) trapped lee waves can occur and (ii) are favored when the surface Richardson number $J=N^{2} /\left(\frac{\partial u}{\partial z}\right)^{2}$ is small. This last result is related to the theoretical fact that the surface absorption of stationary gravity waves increases when $J$ increases. The relation with flow stability is further corroborated by the fact that the trapped lee waves resembles to the $\mathrm{KH}$ modes of instability that exist when $J<0.25$.

For medium mountains some aspects of the theory still hold but need to be adapted, the more intense winds and foehn that occur along the lee side of the mountain having a tendency to increase the surface flow stability. For "initially" small $J$, this can limit the onset of trapped lee-waves, again consistent with the fact that mountain wave surface absorption increases with surface flow stability. For large $J$ the dynamics produces wave breaking on the lee side, destabilizing the flow in the wake of the mountain. In the region where the Richardson number is small, trapped waves develop despite the fact that the surface Richardson number can be quite large, suggesting that the trapped lee waves now result from an absolute instability of the wake.
\end{abstract}

\footnotetext{
${ }^{*}$ Quarterly Journal of the Royal Meteorological Society
} 


\section{Introduction}

Trapped mountains waves generally occur when the vertical profiles of the large scale flow favor the low level confinement of stationary gravity waves. They are sometimes associated with rotors [Doyle and Durran(2002)] and can produce dangerous weather situations near the ground [Keller et al.(2015)]. They also contribute to the low level mountain wave drag [Lott(1998), Georgelin and Lott(2001), Teixeira et al.(2013), ] and still today, it is well established that low level mountain drags improve weather forecast and climate models [Sandu et al.(2015), Pithan et al.(2016), ]. For this reason, there is some debate on which source of low-level mountain drag should be better represented, the results in [Tsiringakis et al.(2017)] suggesting that the trapped lee waves could contribute as much as the blocked flow mountain drag [Lott and Miller(1997), ] or the turbulent subgrid scale orographic drag [1, ]. According to the conventional theory, the low level confinement favoring lee waves can have two dynamical mechanisms. The first attributes the trapped lee waves to free oscillations along a low level density discontinuity. This mechanism has been re-examined recently by [Vosper(2004)] and [Sachsperger et al.(2017)], the first extending the theory to situations where the discontinuity results from a boundary-layer inversion underneath a continuously-stratified atmosphere the latter quantifying analytically the amplitude of the waves in the nonlinear context.

The second mechanism, first identified by [Scorer(1949)], involves the trapping of vertically propagating internal gravity waves between the ground and a turning point aloft. In this theory the Scorer parameter

$$
S=\frac{N^{2}}{U^{2}}-\frac{1}{U} \frac{d^{2} U}{d z^{2}},
$$

plays a central role, $N, U$ and $z$ being the background buoyancy frequency, the background wind and the vertical coordinate respectively. If $S(z)$ decreases with height, due to a diminution of $N$ with altitude for instance, some gravity waves that propagate vertically near above the surface become evanescent aloft. For a given mode with horizontal wave number $k$, this transition occurs at the turning level where $S\left(z_{k}\right)=k^{2}$, and at this level the corresponding wave is reflected downward. The onset of trapped waves then strongly depends to what happen to the reflected waves when they return to the surface. In the initial theory of Scorer (1949), the flow is inviscid and the downward waves are entirely reflected at the surface. In this case a small and discrete number of harmonics survive the multiple interferences between the turning point and the surface, these harmonics dominate the lee waves field. Although the cases where the variations of $S(z)$ are due variations in $N(z)^{2}$ are the most studied ([Jackson et al.(2013)Jackson, Mayr and Vosper, Markowski and Richardson(2011)]), it is clear from Eq. (1) that the variations in $S(z)$ can also be due to the variation of the wind with altitude (examples of trapped lee waves due to increase of the jet stream with altitude in the low troposphere can be found in [Vosper et al.(2013), Teixeira et al.(2013)]). Nevertheless and still according to Eq. (1), the variation of $N$ and $U$ should not be treated on the same footing. To illustrate the difference we can recall that when $U(z)$ becomes very small and even changes sign at a given altitude, $S(z)$ becomes infinite. We are in the presence of a critical level where the waves are often absorbed in the linear case [Booker and Bretherton(1967), ], rather than being entirely reflected as occurs at turning points. The differences become even more important in the nonlinear cases, the mountain waves systematically breaking near that level, the resulting gravity wave mean flow interactions sometimes yielding mountain waves amplification, downslope winds and foehn [Clark and Peltier(1984), Nappo and Chimonas(1992), ]. 
If we now return to the effect of the surface on the lee waves, a major limitation of the inviscid theory is that it neglects the boundary layer effect. For this reason, many numerical studies have included the effect of the boundary layer and shown that the surface friction and the treatment of the turbulent dissipation affect mountain waves ([Bougeault and Lacarrere(1989)], [Richard et al.(1989)]. More recently [Smith et al.(2006)], [Hills et al.(2016)], and [Teixeira (2017)] returned to the basic mechanisms and included some boundary layer absorption in the theory. More specifically, [Smith et al.(2006)] shows that flows that are more unstable near the surface absorb less gravity waves and favor the onset of lee waves. They also noticed that in the presence of a boundary layer the incident flow near the surface can become very small, causing a near critical level situation where the waves can be efficiently absorbed, if the theory of [Booker and Bretherton(1967)] where the critical level is located within the flow applies.

Realizing that the absorption of gravity waves $(\mathrm{GWs})$ by critical levels located at the surface had never been studied, [Lott(2007)] (hereinafter L07) solved this problem theoretically in the viscous case. This paper shows that the wave absorption at the "surface" critical level can be substantial and increases when the surface flow stability increases. This paper also shows that pure reflection only occurs in the inviscid limit and when the surface Richardson number, $J=N^{2} /\left(\frac{\partial u}{\partial z}\right)^{2}<0.25$. To translate how this result affect trapped lee-waves, L07 applied these results to the analysis of the stationary disturbances that can exist in flows with constant stratification and wind that varies like a tanh profile above the surface. This profile was chosen because it has zero wind at the surface, near constant shear immediately aloft and a very smooth transition to constant wind in the far field: it is stationary enough in the viscous case to make the analysis of stationary solutions relevant (at least for reasonably large Reynolds numbers). In such profile, L07 confirmed that GWs absorption increases with $J$ and also that pure trapped modes exist in the inviscid limit when $J<0.25$ : these neutral modes correspond to neutral modes of KH instability found by Drazin (1958) for the same profile in the unbounded case. Latter, these results were confirmed in [Lott(2016)], (hereinafter L16), where an explicit mountain forcing is introduced. The result in L16 also reveals consequences of the near surface critical level dynamics that could not be anticipated from the analysis in L07. It shows that in the presence of a near surface critical levels, the mountain forcing easily triggers strong downslope winds and foehn. Interestingly, downslope windstorms in this case do not result from upper level wave breaking, as often mentions the literature [Durran(1990), ]. In this paper we will question how these downslope winds will modify the background flow in which the trapped waves develop.

One of the more severe limitation of L16 is that it considers free-slip boundary conditions but forces the incident flow to satisfy a no-slip boundary condition far upstream: it does not use the viscous solutions presented in L07 or solutions affected by a turbulent boundary layer as in [Belcher and Wood(1996)]. Although these simplifications are essentially made to simplify the theory they are in part justified by the fact that in the atmosphere the low level wind shears are often due to horizontal gradients in temperature, they do not solely result from boundary layer dynamics: they can extend well above the boundary layer and there, the inviscid dynamics can be applied.

The first purpose of this paper is to describe further the dynamical nature of the trapped lee waves with surface critical level found in L16 and by comparison with a more commonly accepted theory. The second is to analyze if these trapped lee waves still occur in the nonlinear context and with realistic boundary layers. For the first purpose we will compare the nature of trapped modes due to slow low level winds with and without surface critical levels using the L16 the- 
ory. For the second, we will follow [Damiens et al.(2018)Damiens, Lott, Millet and Plougonven] (hereinafter D18), who (i) adapted the theory to higher mountains than in L16, (ii) used in the theory boundary layer depths that gives result comparable to WRF, and (iii) used the WRF model in various idealized configurations and boundary layer specifications. The plan of this paper is as follows. In section 2, the formalism in L16 is extended to higher mountains, it is also applied to flows with and without surface critical levels. This will highlight how dynamically different can be situations where the changes in the background wind only affect the Scorer parameter, to the situation where they both affect the Scorer parameter and induce a near surface critical level. Section 3 then compares the theory with surface critical level and the WRF simulations for small and medium height mountains respectively.

\section{Theory}

\subsection{Continuous variations in $U(z)$}

To analyze the mountain waves produced by a stably stratified shear flow when the incident wind is null at $z=0$, we follow L16 and consider the background flow profiles

$$
U(z)=U_{\infty} \tanh (z / d), N^{2}(z)=\text { const }
$$

incident on a 2-dimensional mountain modeled by the Witch of Agnesi profile:

$$
h(x)=\frac{H}{1+\frac{x^{2}}{2 L^{2}}} .
$$

In (2), $U(z)$ is the background horizontal wind, $N(z)$ the Brunt Vaisala frequency, $z$ the altitude, $d$ is the vertical scale of the shear and $U_{\infty}$ the incident wind maximum amplitude. In (3) $H$ is the maximum mountain height, $L$ its characteristic horizontal length, and $x$ the horizontal coordinate. When scaling time by $N^{-1}$ and distances by $U_{\infty} / N$ the 2-dimensional non-rotating linear dynamics can be expressed in term of a non dimensional vertical velocity, $\bar{w}(\bar{x}, \bar{z})$ of the form,

$$
\bar{w}(\bar{x}, \bar{z})=\int_{-\infty}^{+\infty} f(\bar{k}) \hat{w}_{c}(\bar{k}, \bar{z}) e^{i \bar{k} \bar{x}} d \bar{k}
$$

where overbars denote dimensionless variables, and $\hat{w}_{c}(k, z)$ is a canonical monochromatic solution of "unit" amplitude in the far field which satisfies the dissipative Taylor Goldstein equation,

$$
\frac{d^{2} \hat{w}_{c}}{d \bar{z}^{2}}+\left[\frac{1}{\left(\bar{U}-i \frac{\bar{z}_{k}}{\sqrt{J}}\right)^{2}}-\frac{\bar{U}_{\overline{z z}}}{\bar{U}-i \frac{\bar{z}_{k}}{\sqrt{J}}}-\bar{k}^{2}\right] \hat{w}_{c}=0
$$

In (4) the amplitude term $f(\bar{k})$ is obtained by numerical inversion of the non-linear free-slip boundary condition:

$$
\bar{w}(\bar{x}, \bar{h}(\bar{x}))=[\bar{U}(\bar{h})+\bar{u}(\bar{x}, \bar{h}(\bar{x}))] \frac{d \bar{h}(\bar{x})}{d \bar{x}},
$$

where $\bar{u}(\bar{x}, \bar{z})$ is the horizontal wind disturbance. In (5)-(6)

$$
\bar{U}(\bar{z})=\tanh \left(\frac{\bar{z}}{\sqrt{J}}\right) \text {, and } \bar{h}(\bar{x})=\frac{H_{N}}{1+\bar{x}^{2} / 2 F_{r}^{2}}
$$


where

$$
J=\frac{N^{2} d^{2}}{U_{\infty}^{2}}, H_{N}=\frac{H N}{U_{\infty}}, \text { and } F_{r}=\frac{L N}{U_{\infty}}
$$

are the surface and minimum Richardson number, the non-dimensional mountain height, and the Froude number respectively. Still in (5) the dissipative vertical scale for each harmonics,

$$
\bar{z}_{k}=\frac{\bar{z}_{b}}{2}\left(\frac{1}{F_{r} \bar{k}}+F_{r} \bar{k}\right) .
$$

has been expressed in terms of a global scale $\bar{z}_{b}$, with the first term in parenthesis resulting from Rayleigh drag and Newtonian cooling of coefficient $\bar{z}_{b} / F_{r} / \sqrt{J} / 2$ and the second term from a viscous dissipation which acts in the $x$-direction only of coefficient $\bar{z}_{b} F_{r} / \sqrt{J} / 2$ (for a more thorough discussion of these terms see discussion of Eq. 4 in L16). They are introduced to regularize the critical level dynamics for all the harmonics, i.e. the longer and the shorter ones respectively. Note that $\bar{z}_{k}$ is written differently than in L16 to make clear that when the formula is applied to the dominant wave number $\mathrm{Fr}^{-1}, \bar{z}_{k}$ in (9) is comparable to the dissipative scale $\bar{z}_{b}{ }^{1}$. To construct $\hat{w}_{c}$, L16 uses for each $k$ an exact solution of the inviscid version of (5), $\hat{w}_{\text {inv }}(\bar{k}, \bar{z})$, which is based on hypergeometric functions (see [Lott et al.(1992)]) and (34) in L16, and which asymptotic behaviors are

$$
\begin{gathered}
\hat{w}_{\text {inv }}(\bar{k}, \bar{z}>>1) \approx e^{-\bar{m} \bar{z}} \\
\hat{w}_{\text {inv }}(\bar{k}, \bar{z}<<1) \approx \hat{w}_{\text {mat }}(\bar{k}, \bar{z})=a_{1}(\bar{k}) \bar{z}^{1 / 2-i \mu}+a_{2}(\bar{k}) \bar{z}^{1 / 2+i \mu}
\end{gathered}
$$

where,

$$
\bar{m}=\sqrt{\left|\bar{k}^{2}-1\right|}, \mu=\sqrt{\left|J-\frac{1}{4}\right|}
$$

and where $a_{1}(\bar{k})$ and $a_{2}(\bar{k})$ have analytical forms once the "unit" amplitude condition (10) is satisfied. Also, when $\bar{k}^{2}<1, \bar{m}$ is changed in $-i \operatorname{sign}(\bar{k}) \bar{m}$, where the sign is to ensure upward group speed, and when $J<0.25, \mu$ is changed in $i \mu$. Near the surface, L16 also uses the asymptotic solution of the damped Taylor-Goldstein Equation (5) valid when $\bar{z}<<1$ :

$$
\hat{w}_{\mathrm{Srf}}(\bar{k}, \bar{z})=a_{1}(\bar{k})\left(\bar{z}-i \bar{z}_{k}\right)^{1 / 2-i \mu}+a_{2}(\bar{k})\left(\bar{z}-i \bar{z}_{k}\right)^{1 / 2+i \mu}
$$

where $a_{1}$ and $a_{2}$ are the same as in (11) to ensure convergence toward the "matching" function $\hat{w}_{\text {mat }}$ given in (11) and when $\bar{z} \rightarrow \infty$. Then, following [Bender and Orszag(1978)] we will approximate $\hat{w}_{c}$ by its uniform estimate:

$$
\hat{w}_{c} \approx \hat{w}_{\text {inv }}+\hat{w}_{\text {srf }}-\hat{w}_{\text {mat }} .
$$

The only difference with L16, is that we use this approximation in the inversion of (6),

$$
\int_{-\infty}^{+\infty} f(\bar{k})\left[\hat{w}_{c}(\bar{k}, \bar{h})-\hat{u}_{c}(\bar{k}, \bar{h}) \frac{d \bar{h}}{d \bar{x}}\right] e^{i \bar{k} \bar{x}} d \bar{k}=\bar{U} \frac{d \bar{h}}{d \bar{x}}
$$

rather than using the surface solution in (13) to express $\hat{w}_{c}$ and $\hat{u}_{c}$ in the terms between brackets in (15). This formally permits to consider more elevated ridges than in L16.

\footnotetext{
${ }^{1}$ For consistency, note also that the boundary layer depth $\bar{z}_{B}$ used in L16 is related to the one here by $\bar{z}_{B}=5 \bar{z}_{b}$.
} 


\subsection{Discontinuous variations in $U(z)$}

To contrast our results to the more common case where trapped lee waves are only due to changes in the Scorer parameter and where the ground is not a critical level we also apply our theory to a low level shear zone represented by a two layers flow,

$$
\bar{U}(\bar{z}<\sqrt{J})=\bar{U}_{1}=0.5 \text { and } \bar{U}(\bar{z}>\sqrt{J})=\bar{U}_{2}=1 .
$$

This profile is given in the non-dimensional formalism of subsection (1.1), see also Fig. 1a. For this profile the dissipative Taylor Goldstein equation takes the form,

$$
\frac{d^{2} \hat{w}_{c}}{d \bar{z}^{2}}+\left(\frac{1}{\left(U_{j}-i \bar{z}_{k}\right)^{2}}-\bar{k}^{2}\right) \hat{w}_{c}=0
$$

where the $j=1,2$ index indicates the layer of interest. Also, the continuity of pressure and the uniqueness of the material displacement at the altitude $\bar{z}=\sqrt{J}$ require that

$$
\left[\left(U_{j}-i \bar{z}_{k}\right) \frac{d \hat{w}_{c}}{d \bar{z}}\right]_{j=1}^{j=2}=0, \text { and }\left[\frac{\hat{w}_{c}}{U_{j}}\right]_{j=1}^{j=2}=0
$$

respectively. If we then define vertical "wavenumbers" as

$$
\bar{m}_{j}=\epsilon \sqrt{\bar{k}^{2}-\frac{1}{\left(U_{j}-i \bar{z}_{k}\right)^{2}}}
$$

where the sign $\epsilon= \pm 1$ is chosen so that $\Re\left\{\bar{m}_{j}\right\}>0$, the canonical solution writes,

$$
\hat{w}_{c}=e^{-\bar{m}_{2}(\bar{z}-\sqrt{J})} \text { and } \hat{w}_{c}=\left(\frac{1}{4}+\frac{\bar{m}_{2}}{\bar{m}_{1}}\right) e^{-\bar{m}_{1}(\bar{z}-\sqrt{J})}+\left(\frac{1}{4}-\frac{\bar{m}_{2}}{\bar{m}_{1}}\right) e^{+\bar{m}_{1}(\bar{z}-\sqrt{J})},
$$

for $\bar{z}>\sqrt{J}$ and $\bar{z}<\sqrt{J}$, respectively (see also [Teixeira et al.(2013)]).

\subsection{Inviscid result}

To show the fundamental dynamical differences between the two profiles we next search the neutral solutions that exist in both the inviscid case and when the surface is flat.

When the variations in $U(z)$ are continuous, L07 shows that the dynamics is intimately related to the absorptive properties of the near-surface critical level. To expose them in a concise way we can use the near surface inviscid solution in (11) and express the wave momentum flux

$$
F^{z}=\frac{\hat{u}_{c} \hat{w}_{c}^{*}+\hat{u}_{c}^{*} \hat{w}_{c}}{4}=\left\{\begin{array}{l}
\frac{\mu}{2 \bar{k}}\left(\left|a_{1}\right|^{2}-\left|a_{2}\right|^{2}\right) \quad \text { when } \quad J>0.25, \\
\frac{\mu}{2 \bar{k}}\left(a_{1} a_{2}^{*}+a_{1}^{*} a_{2}\right) \quad \text { when } \quad J<0.25 .
\end{array}\right.
$$

L07 showed that for fixed $a_{1}$, the inviscid solution (11) can be matched to dissipative solutions that satisfy the surface boundary condition, a matching that determines $a_{2}$. It also shows that $\left|a_{2}\right|<\left|a_{1}\right|$ when $J>0.25$, which implies $F^{z}>0$ according to (21): the upward waves dominate the downward propagating ones. The pure surface reflection which is at the basis of the classical trapped lee wave theory [Scorer(1949), ] cannot be satisfied. L07 also shows that the ratio $\left|a_{2} / a_{1}\right|$ tend to decrease when $J$ increases, which in lee waves theory translate into 
the fact that the wave field decay more rapidly with downstream distance when $J$ increases [Smith et al.(2006), ]. L07 also shows that these results still hold in the inviscid limit. When $J<0.25 \mathrm{~L} 07$ also shows that for fixed $\left|a_{1}\right|$ one can have $\left|a_{2}\right|=0$ in the inviscid limit. In this case $F^{z}=0$ according to (21) which means that the reflection is total and trapped lee waves can exist. To find them, the near surface analysis in (11) is nevertheless not sufficient. We have to find if there are indeed solutions of the global inviscid problem that satisfies $a_{2}=0$ near $z=0$. L07 also shows that such solution do exist, they correspond to half of the branch of the neutral modes of KH instabilities found by [Drazin(1958)] (see his Eq. 25). In our definition of the non dimensional parameters it corresponds to the modes for which

$$
\bar{k}^{2}=\frac{1-\sqrt{1-4 J}}{2 J}
$$

This dispersion curve is shown in Fig. 1b.

When the variations in $U(z)$ are discontinuous, the dynamics is almost as in [Teixeira et al.(2013)], and the onset of trapped modes correspond to wave numbers for which $\hat{w}_{c}(z=0)=0$, a condition that imposes,

$$
4 \tanh \left(\bar{m}_{1} \sqrt{J}\right)=-\frac{\bar{m}_{1}}{\bar{m}_{2}} .
$$

In the inviscid case, the only wave numbers that satisfy this condition are those for which $1<\bar{k}<2$ and when:

$$
4 \tan \left(\sqrt{J} \sqrt{4-\bar{k}^{2}}\right)=-\frac{\sqrt{4-\bar{k}^{2}}}{\sqrt{\bar{k}-1}} .
$$

The corresponding dispersion curves are shown in Fig. 1b. It shows that pure trapped waves occur for much "deeper" low level shear layer, the onset of trapped waves being now conditional to $J>\pi^{2} / 12 \approx 0.822$ rather than $J<0.25$ in the continuous case. We also see that when $J$ increases the number of trapped modes increases, this number being given by the resolution of (24) when $\bar{k}=1$, e.g. by the number $n$ satisfying

$$
\frac{\pi}{2}+n \pi<\sqrt{3 J}<\frac{3 \pi}{2}+n \pi
$$

\subsection{Forced dissipative results}

To include an explicit forcing, we next proceed numerically as in L16, discretize in the spectral space the Fourier integral in (4), taking for $\hat{w}_{c}(14)$ in the continuous case or (20) in the stairway case. In both, cases, $f(\bar{k})$ is determined through the numerical inversion of the nonlinear boundary condition (15). In the discrete space this inversion is a matrix inversion that only converges with increasing resolution when there is dissipation and because the surface boundary condition becomes singular in the inviscid case when there are resonant modes. In this section, we take a domain of length 500, spanned by 2048 equally spaced points, the boundary layer parameter $z_{b}=0.01$, and the non-dimensional mountain height $H_{N}=0.1$. We also take $F r=2$, which means that waves with wavenumbers around $F r^{-1}=0.5$ will be forced. This value is sufficiently near the lower bound of the dispersion curves in Fig. 1 b to ensure that some trapped waves will be well excited.

The two panels in Figs. 1c) and 1d) show the vertical velocities around the center of the shear layer in $\bar{z}=\sqrt{J} / 2$, for the continuous and discontinuous profiles respectively. As expected 
from L16, one sees that in the presence of a surface critical level, trapped lee waves develop more easily when the flow is unstable, i.e. for small $J$ 's, whereas it is the other way round when the background flow is discontinuous. In the later case, trapped waves develop more easily when the parameter $J$ becomes larger than one, consistent with the inviscid results for the stairway case in section 2.3). These results corroborate that reasoning in terms of trapping only can be misleading. If the surface critical level dynamics is neglected, increasing the depth of the shear increases the depth of the low level wave duct: more and more modes can be trapped within it. When including reasoning in terms of shear flow stability, the surface critical level dynamics becomes central, and, when the depth of the shear zone decreases, the surface critical level absorption decreases, which favors trapped lee waves.

\section{$3 \quad$ Fully nonlinear simulations}

\subsection{Experimental setup}

To test if the relation between flow stability and trapped mountain waves still hold in the fully non linear context, we next follow D18 and use the WRF model in the 2D mountain flow configuration available on line [Skamarock et al.(2005), ]. In all the simulations presented we consider a mountain of length $L=1.1 \mathrm{~km}$ which height typically varies between $50 \mathrm{~m}$ and $500 \mathrm{~m}$. To capture well the gravity waves dynamics the model domain is $80 \mathrm{~km}$ long and $9 \mathrm{~km}$ high, and both directions are discretized by 800 x 450 equally spaced points respectively. These yield an horizontal resolution of $\Delta x=100 \mathrm{~m}$ and a vertical resolution of $\Delta z=20 \mathrm{~m}$, both are near an order of magnitude smaller than the mountain dimension. These rather high resolutions force to use a time step of $\Delta t=0.5 \mathrm{~s}$, they are necessary to guarantee that the near surface critical level dynamics is well captured, and we verified that our results are not much changed when these resolutions and domain sizes are changed by a factor 2 . To allow gravity waves to propagate away from the domain without lateral and upper boundary reflections we use open lateral boundary conditions and introduce a $6 \mathrm{~km}$ high damping layer below the domain lid. To impose a constant buoyancy frequency, and because the WRF model is fully compressible we take an isothermal atmosphere with $T_{0}=288 \mathrm{~K}$. This results in a constant Brunt-Vaisala frequency $N$, with $N^{2}=\frac{g}{\theta_{0}} \frac{d \theta_{0}}{d z}=\frac{\kappa g^{2}}{R T_{0}}=3.3210^{-4} \mathrm{~S}^{-2}$, and where $\kappa, R$ and $g$ have their usual earth values, and $\theta_{0}$ is the background potential temperature. The background wind is defined by (7) with $U_{\infty}=10 \mathrm{~m} \mathrm{~s}^{-1}$. In this setup the froude number value $F r=2$, as in section 2 and will not vary, the sensitivity to the surface Richardson number $J$ will be analyzed by changing the value of the shear layer depth $d$, and that to the mountain height by varying the mountain height $H_{N}$. In most case, and except when specified the results are shown after $4 \mathrm{~h}$ of integration, that is much longer than the advective time-scale $\frac{L}{U_{\infty}} \approx 100 \mathrm{~s}$, and sufficiently long for the trapped lee waves to develop downstream.

As the theory in Section 2 is based on an free-slip treatment of the lower boundary, we will first present WRF simulations where the constant diffusion coefficients are put to zero and where the surface boundary condition is free-slip. Nevertheless, this does not necessarily make the simulations inviscid because irreversible or diabatic processes certainly occur near the surface : it happens that the WRF dynamical core is stable and dissipative enough to handle these processes (see discussion in D18). When we return to the theory, it is a priory difficult to say how these irreversible processes can affect the wave dynamics. Nevertheless, as the theory has a free parameter to represent dissipation, the boundary layer scale $\bar{z}_{b}$, we can vary it and 
try to identify a value for which there is a match between the nonlinear simulations and the theory. In D18, it was found that a dependance in $\sqrt{J}$ helped the comparison with WRF and we take here

$$
\bar{z}_{b} / \sqrt{J}=0.005 .
$$

We well keep this value in all the following experiments with the theoretical model.

To extent even further the significance of our results, we have also used WRF with two boundary layer schemes that have been extremely validated by the community, the Yonsei University Planetary Boundary Layer scheme (hereinafter YSU BLYR, [Hong et al.(2006)]) and the Mellor Yamada scheme (hereinafter MY BLYR, [Mellor and Yamada(1982)]). Although the two schemes have fundamental differences, the YSU BLYR is based on the nonlocal diffusion concept of [Troen and Mahrt(1986)] whereas the MY calculate local diffusion coefficients with amplitude controlled by the amplitude of a turbulent kinetic energy evaluated at each time step, we do not expect them to behave very differently in all the dry and initially convectively stable cases we consider. The intention here is more to test robustness using well known schemes, and although the two schemes may well regularize the convectively unstable regions produced by the waves quite differently. In these two configurations where the interaction with the surface is more thoroughly taken into account we force the potential temperature of the surface to equals the background potential temperature, $\theta_{s}(x)=T_{0} e^{\kappa h(x) / H_{0}}$, where the characteristic height $H_{0}=R T_{0} / g$, whereas the roughness length has its default value $z_{0}=0.1 \mathrm{~m}$.

\subsection{Low mountain}

The left panels in Fig. 2 show the vertical velocity fields produced by the theoretical model for three different values of $J$, and the right panels show the same fields evaluated with the free-slip WRF simulations. In all panels, the results are shown using non-dimensional variables to ease comparison with theory and the mountain maximum height $H_{N}=0.1$. The first striking result is that the theory and WRF compare very well qualitatively and quantitatively. This is maybe the most important result of this work: the theory is based on a linear approximation that is often questionned in the presence of critical levels [Lott and Teitelbaum(1992), Dörnbrack and Nappo(1997)].

If we return to the fields themselves, we see that for $J=3$ in Figs. $2 \mathrm{a}$ and $2 \mathrm{~b}$, the vertical velocity field is largely dominated by vertically propagating mountain waves, the sign of the constant phase lines slope being opposite to that of the incident wind. In both models, the wave field substantially extents downstream of the ridge, which is characteristic of non-hydrostatic effect when the background flow is uniform $[\mathrm{Queney}(1947)$, ] and the Froude number near $F r=1$. Here the interpretation holds above the shear layer, i.e. when $\bar{z}>\sqrt{J}$. Still for $J=3$, note that in WRF, the wave amplitude is a little smaller than in the theory, but this difference could be sorted out by moderately increasing the boundary layer depth $z_{b}$ in the latter (not shown).

If we now look at smaller values of $J$, we see that for $J=0.75$ the theory and WRF in Figs. $2 \mathrm{c}$ and $2 \mathrm{~d}$ still predict comparable fields, trapped lee waves start to appear, although they are still quite small in both models. Note that now, it is the theory that predicts smaller amplitude wave field than WRF, which could suggest that we should now decrease the boundary layer depth $z_{b}$ in the theory. As this amplitude factor changes from one experiment to another this indicates that the relation between $z_{b}$ and $J$ we have taken here and in D18, should eventually 
be improved or made dependent on the Froude number or on $H_{N}$. Nevertheless as this does not affect our interpretation of the results we prefer to keep the relation (26).

In both models, and for even smaller values of $J$, (i.e, $J=0.16$ ) we see again that WRF and theory give comparable results, but now both present a well defined train of lee waves, extending downstream down to more than $50 \mathrm{~km}$. These trains of lee waves are characterized by almost vertical phase lines, the vertical velocity fields being essentially confined within the shear layer (see $U(z)$ in the left part of each panel). A difference is that in the theory, the train of lee waves seems to dissipate faster than in WRF, again this can be easily sorted out by decreasing further $z_{b}$ in the theory, an issue that is not critical for our interpretation of the results. A more interesting aspect concern the horizontal wavelength. We see that in both models it is near equals $\bar{\lambda} \approx 5$, which corresponds to $\bar{k} \approx 1.05$, whereas the corresponding $\mathrm{KH}$ mode in $(22)$ has $\bar{k} \approx 1.10$ for $J=0.16$. If we remember that the Drazin solutions are confined to the shear layer and have only one node for the vertical velocity, the inflection point of the background wind (here $z=0$ ), it is clear that the wave field downstream in Figs. 2e and $2 \mathrm{f}$ has structure of a neutral mode of $\mathrm{KH}$ instability.

As the central objective of this paper is focused on the trapped lee-waves development, we next choose a representation that emphasize them. For this purpose, the Fig. 3 shows the horizontal variations of the vertical velocity at the altitude $\bar{z}=1$. The panels in the left column are for the theory (Fig. 3a), and are consistent with the results in L16, which establishes that more unstable flows favor trapped lee waves. In L16 nevertheless dissipations are almost absent, which makes that the lee waves for $J<0.25$ are almost pure trapped lee waves, their amplitude do not decay downstream, the mountain exciting a neutral mode of $\mathrm{KH}$ instability. Also, in L16, the boundary layer depth does not increase with $J$, which here absorbs even further the waves when $J$ increase: this makes that in Fig. 3a) almost no waves are present when $J>1$.

A striking result is that WRF reproduces well this dependence with $J$, in the free-slip case which we already discussed but confirm here more systematically. What is more interesting is that with more sophisticated boundary layer parameterization schemes in Figs. 3c and 3d the transition from more unstable flows with more trapped waves to more stable flows with less trapped waves still hold. As said in the model description, our choice was to use well tuned and stable schemes to confirm our findings beyond the free slip assumption, rather than to discuss with details the differences. What can be said here is that imposing a surface drag via similarity theory, what fundamentally do both the MY-BLYR and the YSU-BLYR schemes, does not fundamentally change the absorptive properties of the waves near the surface compared to the inviscid case, and hence the downstream development of the trapped lee-waves. A reason is probably that our background winds are small near the surface, and the roughness length is also quite small, two reasons that make the frictional drag small and the results quite consistent with the free-slip cases.

\subsection{Medium height mountain}

To test if some of our results hold for higher mountains, we next present experiments with $H_{N}=0.7$, that is $H=404 \mathrm{~m}$ in dimensional units. The results for the horizontal profiles of the vertical velocity in Fig 4 confirm that some aspects of the theory are still relevant. The most remarkable one is that trapped waves do occur in most experiments, they all have horizontal wavenumber around $\bar{k}=1$, their spatial structure also resemble to KH neutral modes (not shown). Although there is an overall tendency for the simulations with small $J$ to produce 
trapped waves propagating over longer distances downstream, some remarkable differences start to occur.

The first is that for small $J$ and except when the MY BLYR scheme is used, the first minimum in vertical velocity located immediately downstream of the ridge becomes substantially larger in amplitude compared to the oscillations associated with the lee wave. This follows that enhanced non-linearities increase downslope winds, a behavior already analyzed in D18. We will not discuss it further here, essentially because this effect is present in the theory in Fig. 4 for $J=0.16, J=0.23$, and $J=0.3$, as well as in the free slip case, and to a lesser extent when using the YSU BLYR. Note that this enhancement of downslope winds is almost absent with MY-BLYR, indicating that turbulent parameterizations affect seriously the downslope winds. As we shall see with more details below this impacts in return the development of the trapped lee waves: at small $J$ and with MY-BLYR in Fig. 4d), the downslope winds are not as intense as with the other BLYR, which seems to favor the downstream development of the lee waves.

The second difference is more directly related to the lee wave downstream extension. In some cases it can almost disappear locally at small $J$ (for instance in the free-slip case in Fig. 4b when $J=0.16$ between $1<\bar{x}<30$ ). Conversely lee waves can develop downstream more substantially than in the theory, as occur quite systematically in WRF when $J>1$. To test if these changes challenge our interpretation of trapped lee waves in terms of stability, we next analyze with more details the cases with the two extreme values $J=0.16$ and $J=5$.

The results above suggest that when $H_{N}$ approaches 1 the interpretation in term of upstream flow surface stability becomes incomplete. Interestingly for such values of $H_{N}$, we also know that strong downslope winds and low level wave breaking can occur. These certainly change the large-scale flow downstream and hence the local properties of the flow in which the trapped waves develop. To substantiate this relation between local flow stability and trapped waves, we next use the fact that the lee wave fields shown in Fig. 4 have rather well defined horizontal wavelength: we can make a separation between the "large-scale flow" and the waves by averaging fields over horizontal distances that compare with the horizontal wavelength. In the following, as the trapped waves have wavelength that are always near and below $3 \mathrm{~km}$, we will apply a top hat horizontal filter with a fixed length of $3 \mathrm{~km}$ to extract the large scale, but we found little sensitivity when moderately increasing the horizontal length of the filter.

For $J=0.16$ the top panels in Fig. 5 show the temporal evolution of the altitude of the "unfiltered" isentrope $\theta=291 \mathrm{~K}$, and which upstream altitude is almost equal to $H$. The time evolution in the free slip case in Fig. 5a shows the development of a moderate Foehn downslope, the region where the isentrope altitude is below the mountain top extents with time to reach near $x=20 \mathrm{~km}$ after $t=240 \mathrm{~min}$. This region of moderate Foehn does not end by an abrupt hydraulic jump, consistent with the fact that strong Foehn are favored when $J$ is large (see L16, D18). In term of trapped waves, what is interesting is that the oscillations with wavelength below $3 \mathrm{~km}$ are stationary, as expected for mountain waves, but begins downstream the Foehn region. The panel below in Fig. 5d shows the raw fields of potential temperature altitude at $t=240 \mathrm{~min}$, superimposed onto the "large scale" Richardson number,

$$
R_{i f}=\frac{g}{\theta_{f}} \frac{\partial \theta_{f}}{\partial z} /\left(\frac{\partial u_{f}}{\partial z}\right)^{2}
$$

where $\theta_{f}$ and $u_{f}$ are the filtered field of potential temperature and horizontal wind respectively. Here we see that the region where the trapped waves are present are also characterized by regions where $R_{i f}$ is small near the surface, and which are quite far downstream of the ridge. 
In Figs. 5b one sees that an effect of the YSU-BLYR layer parameterization is to reduce the Foehn intensity, the descent of the $\theta=291 \mathrm{~K}$ surface downstream is less pronounced than in the inviscid case, and the flow near the surface still has $R_{i f}<0.25$, as indicates the green curves and at least until the $x=20 \mathrm{~km}$. Above this region substantial small scale oscillations do occur, whereas beyond $x=20 \mathrm{~km}$, the surface flow is stabilized and the isentropes no longer present small scale oscillations. This correspondence between surface flow stability and trapped waves is also found when the MY BLYR scheme is used (see Figs. 5c-5f), in this case also the scheme reduces the foehn amplitude, the flow stays unstable on the lee-side of the ridge, it even stays so down to at least $x=40 \mathrm{~km}$ and trapped waves are present almost everywhere in this zone. In all cases, we see that the correspondence between trapped waves and the near surface flow stability continues to hold.

The differences between the theory and the WRF are even more pronounced when the initial background flow is stable as show the simulations with $J=5$ in Fig. 6. First, in the free slip case, the $\theta=291 \mathrm{~K}$ surface in Fig 5 a presents a pronounced foehn immediately downstream of the ridge, followed by an abrupt transition beyond which trapped lee-waves develop. Again the trapped lee waves field develops in time, but is stationary: the first pronounced crest at around $x=10 \mathrm{~km}$ for instance stays almost at the same place, and this is true for the other crests. The fields of large scale flow stability and isentropic surface in Fig $5 \mathrm{~d}$ show that on the lee side, the dynamics has not much modified the surface flow stability (the Richardson number near $z=0$ is still much larger than 1 ), but produced in the mountain wake a large unstable zone that extent with time. We see in Fig. 5d that this unstable zone is also where the trapped wave signal is the most pronounced. According to this result, it seems reasonable to suggest that the trapped waves are again related to an instability triggered by the mountain, as suggests the stationarity of the lee wave.

To support this, one needs to verify that where the flow is unstable the large-scale wind is also small, and following that (i) KH instabilities have a phase speed near the large-scale wind value in the region where they are produced, and (ii) mountains force stationnary waves. This is verified in Fig. 7a, which shows profiles from filtered filelds at $x=13 \mathrm{~km}$. In it, we see that in the region of instability, the large scale wind is quite small, the near unstable modes, if they exist, are likely to have small phase speed and to be excited by an orographic forcing. The results from other boundary layer parameterizations in Figs. 6b-6e and Figs. 6c-6f somehow corroborate these results. In all of them, trapped waves develop whereas the surface stability stays strong. They seem associated to the unstable regions located well above the surface in the wake of the mountain, and in all cases the large scale winds in the region where the Richardson number is small are quite small (Figs. 7b and 7c). A marked difference is that between the free slip case and the cases with BLYR parameterization the horizontal wavelength of the waves is quite different, an other is that the intensity of the foehn is much less pronounced with the BLYR parameterizations than in the free slip case.

\section{Conclusion}

Using theory and WRF simulations with various boundary layer parameterization, this paper has shown that the onset of trapped lee waves is strongly linked to the stability property of the background flow. This complement our conventional view of trapped lee waves where the dynamics is mainly explained in term of variations of the Scorer parameter. We believe that 
this view becomes mandatory when the variations in the Scorer parameter are due to variations of the incident wind rather than of stratification, and when the incident wind is quite small near the surface. For small mountains, it happens that the theory in L16 explains well this dependence. Small surface Richardson numbers result in large gravity wave surface reflections and favor the trapped wave development. For medium height mountain, the nonlinear dynamics in the wake of the mountain modify the large scale flow, and therefore the background properties in which the trapped waves develop. For small $J$, non-linearities yield in an increase of the surface Richardson number near the surface and the trapped waves are attenuated. For large $J$, strong downslope windstorms happen and yield mixing that destabilizes the flow in the wake of the mountain. In this case, we attribute the onset of trapped waves to the triggering of small phase speed unstable modes.

Despite the fact that there is some coherency in our results concerning the links between trapped waves and instabilities, it is important to note that the boundary layer parameterizations have strong quantitative impacts. These impacts are extremely evident in the medium height mountain simulation (section 3.3). In this case and when the background flow is unstable near the surface $(J=0.16)$, the dynamics stabilizes the surface flow in the free slip case, whereas it does not affect it much when the MY-BLYR scheme is used. This impacts the location of the trapped waves. For very stable cases $J=5$, the dynamics produce strong foehn in the free-slip case, whereas the Foehn is less intense with the YSU and MY boundary layer parameterizations. Again the location of the trapped waves is affected in return. These results clearly illustrate that the boundary layer parameterizations have strong impacts on mountain wave dynamics, at least when the incident wind present strong shears in the low troposphere. In essence, the results obtained illustrate again the significance of low level wind shears and stability on mountain flow dynamics, something often noticed in the context of trapped lee waves [Reinecke and Durran(2009), Georgelin and Lott(2001)]. In this context it is interesting to note that there are many field experiments where upstream soundings show low level shears [Doyle et al.(2011), Sheridan et al.(2007)] generally related to advancing fronts [Lothon et al.(2003)], when the low level shear is associated with thermal wind balance.

\section{Acknowledgements:}

This work was supported by the ANR/JPI-Climate/Belmont Forum project GOTHAM (ANR15-JCLI-0004-01).

\section{References}

[Belcher and Wood(1996)] Belcher S, Wood N. 1996: Form and wave drag due to stably stratified turbulent flow over low ridges. Quarterly Journal of the Royal Meteorological Society 122(532): 863-902.

[1] Beljaars ACM, Brown AR, Wood N. 2004: A new parametrization of turbulent orographic form drag. Quarterly Journal of the Royal Meteorological Society 130(599): 1327-1347.

[Bender and Orszag(1978)] Bender C, Orszag S. 1978. Advanced mathematical methods for scientists and engineers. McGraw-Hill. 
[Booker and Bretherton(1967)] Booker J, Bretherton F. 1967. The critical layer for internal gravity waves in a shear flow. Journal of Fluid Mechanics 27(03): 513-539.

[Bougeault and Lacarrere(1989)] Bougeault P, Lacarrere P. 1989. Parameterization of orography-induced turbulence in a mesobeta-scale model. Monthly Weather Review 117(8): 1872-1890.

[Clark and Peltier(1984)] Clark T, Peltier W. 1984. Critical level reflection and the resonant growth of nonlinear mountain waves. Journal of the Atmospheric Sciences 41(21): 31223134 .

[Damiens et al.(2018)Damiens, Lott, Millet and Plougonven] Damiens F, Lott F, Millet C, Plougonven R. 2018. An adiabatic foehn mechanism. Quarterly Journal of the Royal Meteorological Society : 1-13doi:https://doi.org/10.1002/qj.3272.

[Dörnbrack and Nappo(1997)] Dörnbrack A, Nappo CJ. 1997. A note on the application of linear wave theory at a critical level. Boundary-Layer Meteorology 82(3): 399-416.

[Doyle and Durran(2002)] Doyle JD, Durran DR. 2002. The dynamics of mountain-waveinduced rotors. Journal of the Atmospheric Sciences 59(2): 186-201.

[Doyle et al.(2011)] Doyle JD, Gabersek S, Jiang Q, Bernardet L, Brown JM, Dornbrack A, Filaus E, Grubisic V, Kirshbaum DJ, Knoth O, Koch S, Schmidli J, Stiperski I, Vosper S, Zhong S. 2011. An intercomparison of t-rex mountain-wave simulations and implications for mesoscale predictability. Monthly Weather Review 139: 2811-2831, doi: http://dx.doi.org/10.1175/MWR-D-10-05042.a.

[Drazin(1958)] Drazin P. 1958. The stability of a shear layer in an unbounded heterogeneous inviscid fluid. Journal of Fluid Mechanics 4(2): 214-224.

[Durran(1990)] Durran DR. 1990. Mountain waves and downslope winds. AMS Meteorological Monographs 23: 59-83.

[Georgelin and Lott(2001)] Georgelin M, Lott F. 2001. On the transfer of momentum by trapped lee waves. case of the iop3 of pyrex. Journal of the Atmospheric Sciences 58: $3563-3580$.

[Hills et al.(2016)] Hills MOG, Durran DR, Blossey PN. 2016. The dissipation of trapped lee waves. part ii: The relative importance of the boundary layer and the stratosphere. Journal of the Atmospheric Sciences 73(3): 943-955.

[Hong et al.(2006)] Hong SY, Noh Y, Dudia J. 2006. A new vertical diffusion package with an explicit treatment of entrainment processes. Monthly Weather Review 134(9): 2318-2341.

[Jackson et al.(2013)Jackson, Mayr and Vosper] Jackson P, Mayr G, Vosper S. 2013. Dynamically-driven winds. In: Mountain weather research and forecasting, Springer, pp. $121-218$.

[Keller et al.(2015)] Keller T, Trier S, Hall W, Sharman R, Xu M, Liu Y. 2015. Lee waves associated with a commercial jetliner accident at denver international airport. Journal of Applied Meteorology and Climatology 54(7): 1373-1392. 
[Long(1953)] Long RR. 1953. Some aspects of the flow of stratified fluids; 1. a theoretical investigation. Tellus 5: 42-58.

[Lothon et al.(2003)] Lothon M, Druilhet A, Benech B, Campistron B, Bernard S, Sad F. 2003. Experimental study of five foehn events during the mesoscale alpine programme: From synoptic scale to turbulence. Quarterly Journal of the Royal Meteorological Society 129(592): 2171-2193, doi:10.1256/qj.02.30, URL http://dx.doi.org/10.1256/qj.02.30.

[Lott(1998)] Lott F. 1998. Linear mountain drag and averaged pseudo momentum profiles in the presence of trapped lee waves. Tellus 50A: 12-25.

[Lott(2007)] Lott F. 2007. The reflection of a stationary gravity wave by a viscous boundary layer. Journal of the Atmospheric Sciences 64(9): 3363-3371.

[Lott(2016)] Lott F. 2016. A new theory for downslope windstorms and trapped mountain waves. Journal of the Atmospheric Sciences 73(9): 3585-3597.

[Lott et al.(1992)] Lott F, Kelder H, Teitelbaum H. 1992. A transition from kelvin-helmholtz instabilities to propagating wave instabilities. Physics of Fluids A 4: 1990-1997, doi: http://dx.doi.org/10.1063/1.858368.

[Lott and Miller(1997)] Lott F, Miller MJ. 1997. A new subgrid-scale orographic drag parametrization: Its formulation and testing. Quarterly Journal of the Royal Meteorological Society 123(537): 101-127, doi:10.1002/qj.49712353704.

[Lott and Teitelbaum(1992)] a stratified shear flow: instabilities and gravity waves. Geoph. Astr. Fluid Dyn. 66: 133-167.

[Markowski and Richardson(2011)] Markowski P, Richardson Y. 2011. Mesoscale meteorology in midlatitudes, vol. 2. John Wiley \& Sons.

[Mellor and Yamada(1982)] Mellor G, Yamada T. 1982. Development of a turbulence closure model for geophysical fluid problems. Reviews of Geophysics 20(4): 851-875.

[Nappo and Chimonas(1992)] Nappo C, Chimonas G. 1992. Wave exchange between the ground surface and a boundary-layer critical level. Journal of the atmospheric sciences 49(13): 1075-1091.

[Pithan et al.(2016)] Pithan F, Shepherd TG, Zappa G, Sandu I. 2016. Missing orographic drag leads to climate model biases in jet streams, blocking and storm tracks. Geophysical Research Letters 43: 7231-7240.

[Queney(1947)] Queney P. 1947. Theory of perturbations in stratified currents with application to airflow over mountain barriers. The University of Chicago Press (Misc. Rep. 23).

[Reinecke and Durran(2009)] Reinecke PA, Durran DR. 2009. Initial-condition sensitivities and the predictability of downslope winds. Journal of the Atmospheric Sciences 66: 3401-3418, doi:http://dx.doi.org/10.1175/2009JAS3023.1.

[Richard et al.(1989)] Richard E, Mascart P, Nickerson E. 1989. The role of surface friction in downslope windstorms. Journal of Applied Meteorology 28(4): 241-251. 
[Sachsperger et al.(2017)] Sachsperger J, Serafin S, Grubišić V, Stiperski I, Paci A. 2017. The amplitude of lee waves on the boundary-layer inversion. Quarterly Journal of the Royal Meteorological Society 143(702): 27-36.

[Sandu et al.(2015)] Sandu I, Bechtold P, Beljaars A, Bozzo A, Pithan F, Shepherd T, Zadra A. 2015. Impacts of parameterized orographic drag on the northern hemisphere winter circulation, journal of advances in modeling earth systems. J. Adv. Model. Earth Syst. 8: 196-211, doi:DOI : 10.1002/2015MS000564.

[Scorer(1949)] Journal of the Royal Meteorological Society 75(323): 41-56.

[Sheridan et al.(2007)] Sheridan PF, Horlacherxi V, Rooney GG, Hignett P, Mobbs SD, Vosper S. 2007. Influence of lee waves on the near surface flow downwind of the pennines. Quarterly Journal of the Royal Meteorological Society 133: 1353-1369, doi:10.100a/2qj.110.

[Skamarock et al.(2005)] Skamarock WC, Klemp J, Dudhia J, Gill D, Barker D, Wang W, Powers J. 2005. A description of the advanced research wrf version 2. Technical report, National Center For Atmospheric Research Boulder Co Mesoscale and Microscale Meteorology Div.

[Smith et al.(2006)] Smith RB, Jiang Q, Doyle JD. 2006. A theory of gravity wave absorption by a boundary layer. Journal of the Atmospheric Sciences 63: 774-781, doi: http://dx.doi.org/10.1175/JAS3631.1.

[Teixeira (2017)] Teixeira MA. 2017. Diagnosing Lee Wave Rotor Onset Using a Linear Model Including a Boundary Layer. Atmosphere 8(1): 5.

[Teixeira et al.(2013)] Teixeira MA, Argaín J, Miranda P. 2013. Drag produced by trapped lee waves and propagating mountain waves in a two-layer atmosphere. Quarterly Journal of the Royal Meteorological Society 139(673): 964-981.

[Troen and Mahrt(1986)] Troen I, Mahrt L. 1986. A simple model of the atmospheric boundary layer; sensitivity to surface evaporation. Boundary-Layer Meteorology 37(1-2): 129-148.

[Tsiringakis et al.(2017)] Tsiringakis A, Steeneveld G, Holtslag A. 2017. Small-scale orographic gravity wave drag in stable boundary layers and its impact on synoptic systems and near-surface meteorology. Quarterly Journal of the Royal Meteorological Society 143(704): 1504-1516, doi:10.1002/qj.3021.

[Vosper et al.(2013)] Vosper S, Wells H, Sinclair J, Sheridan P. 2013. A climatology of lee waves over the uk derived from model forecasts. Meteorological Applications 20(4): 466-481.

[Vosper(2004)] Vosper SB. 2004. Inversion effects on mountain lee waves. Quarterly Journal of the Royal Meteorological Society 130(600): 1723-1748.

\section{acknowledgements}

This publication was partly funded by the ANR/JPI-Climate/Belmont Forum project GOTHAM (ANR-15-JCLI-0004-01). 


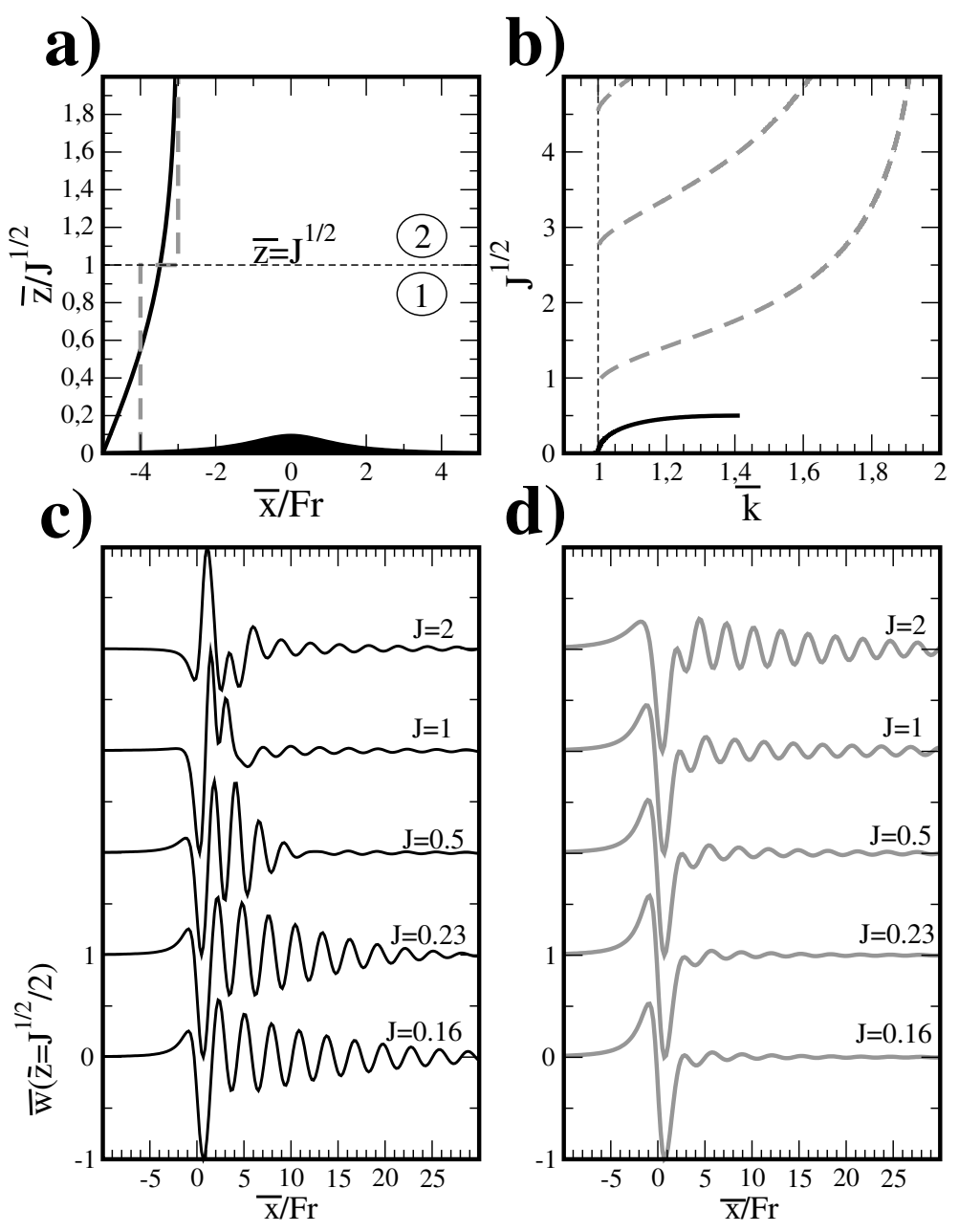

Figure 1: (a) Mountain profile and background wind configurations used in the theory: $\bar{U}$ in tanh (black solid), $\bar{U}$ stairway (grey dashed); (b) Dispersion curves (22) and (24) (same color convention); c) vertical velocity at $\bar{z}=\sqrt{J} / 2$ from the dissipative model when $\bar{U}$ is in tanh: d) Same as c) but when $\bar{U}$ is stairway. 

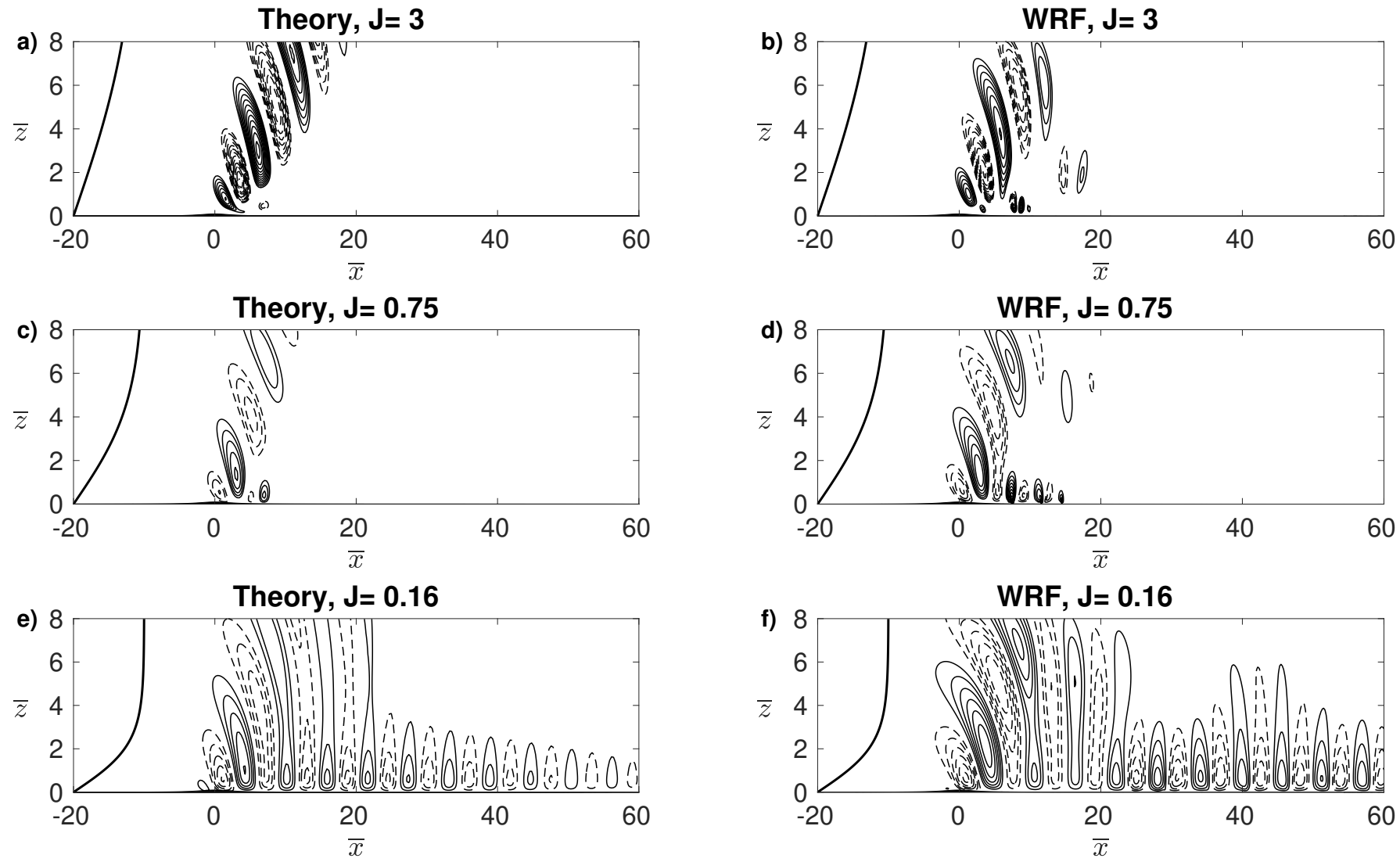

Figure 2: Non dimensional vertical velocity from the theoretical model and from free-slip WRF simulations. In all panels the background wind is shown on the left, $H_{N}=0.1$, and the contour interval is $0.001 / \sqrt{J}$. 

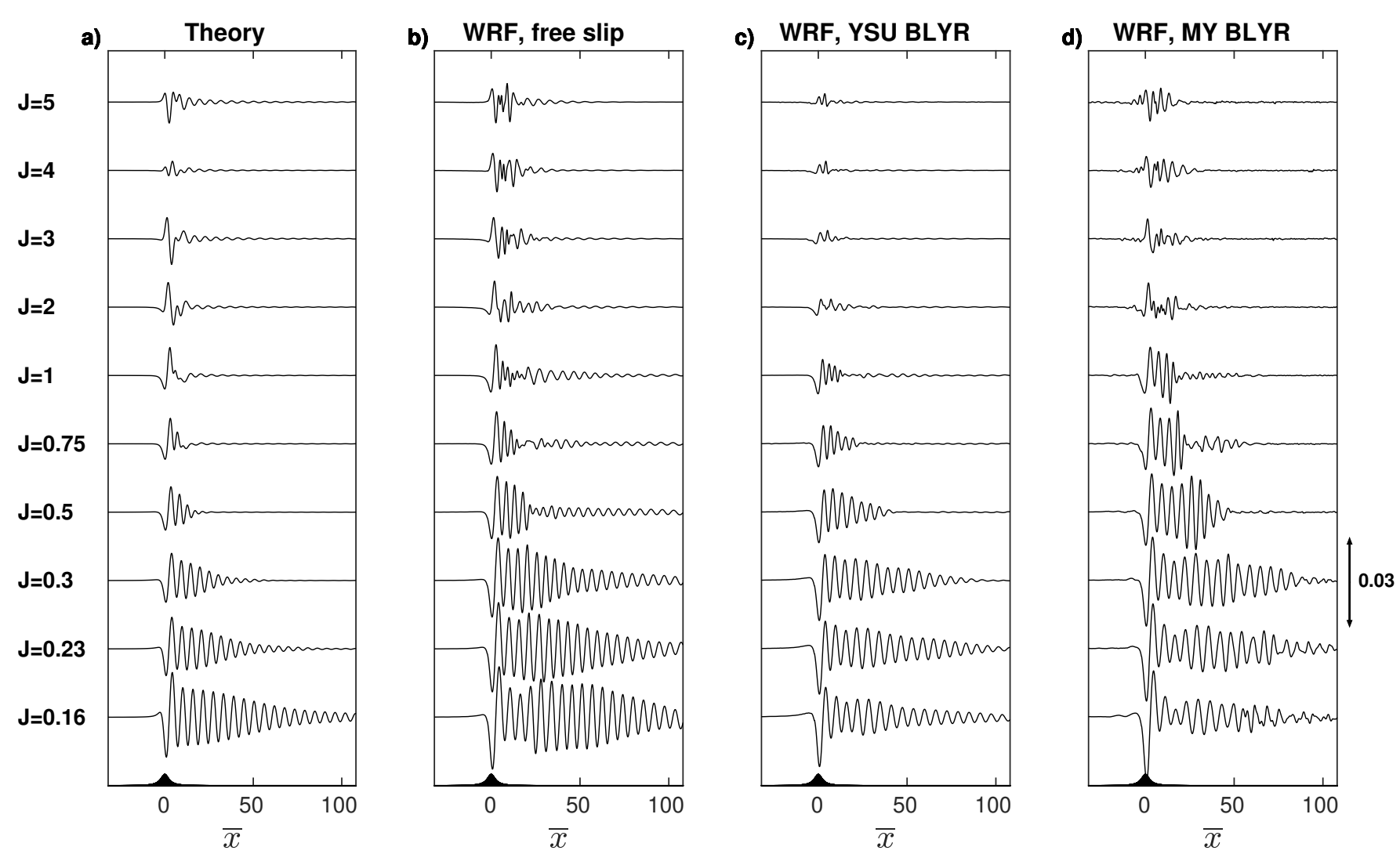

Figure 3: Horizontal profiles of non dimensional vertical velocity at $\bar{z}=1$, with $H_{N}=0.1$ and for various $J$. All curves have the same vertical scale given by the arow on the right. 

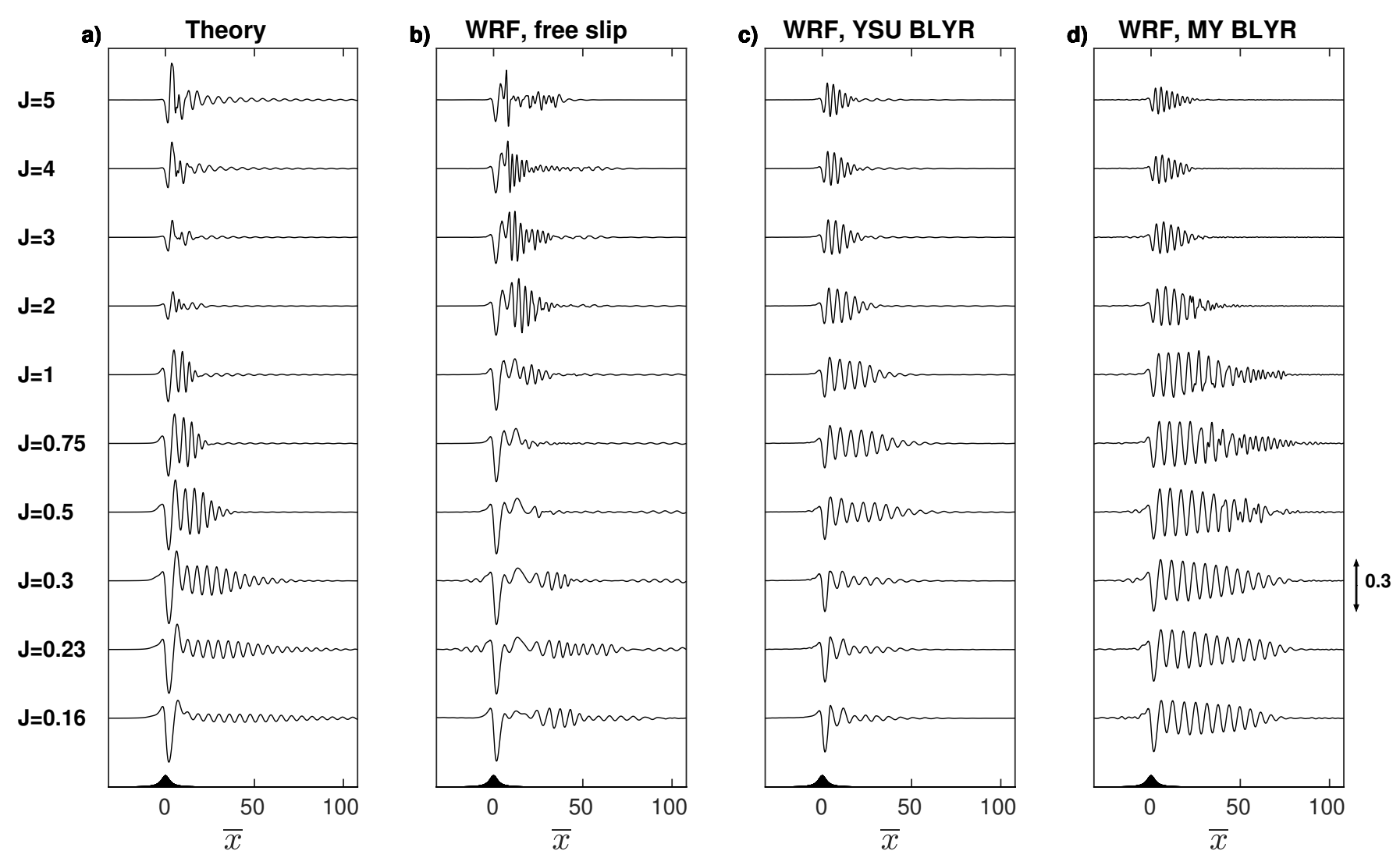

Figure 4: Same as Fig 3 but for $H_{N}=0.7$. 
a)

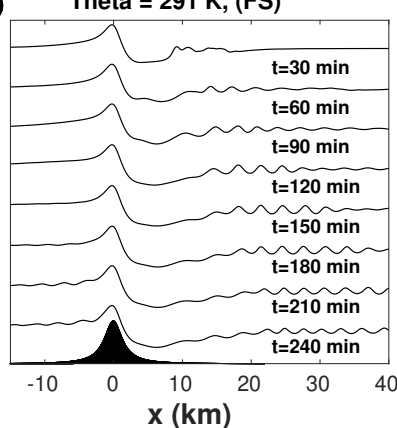

d) $R_{i}$ and theta with $\mathrm{J}=\mathbf{0 . 1 6}$, (FS)

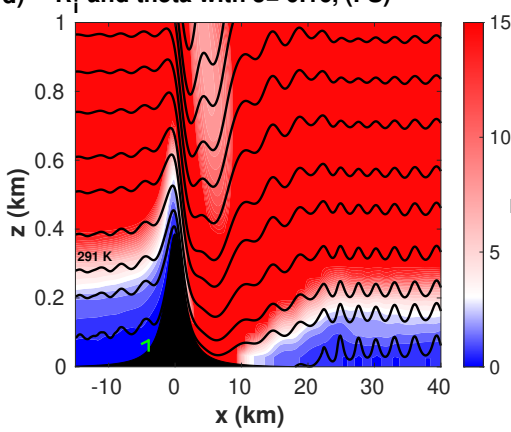

b)

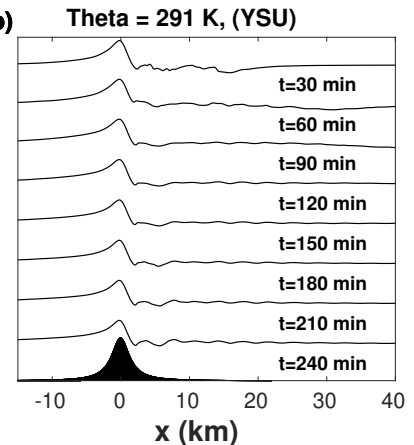

e) $R_{i}$ and theta with $\mathrm{J}=0.16$, (YSU)

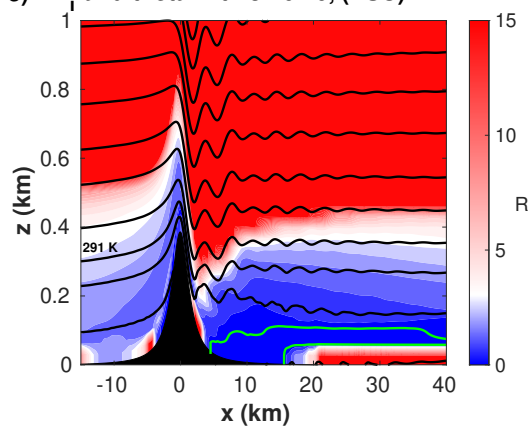

c)

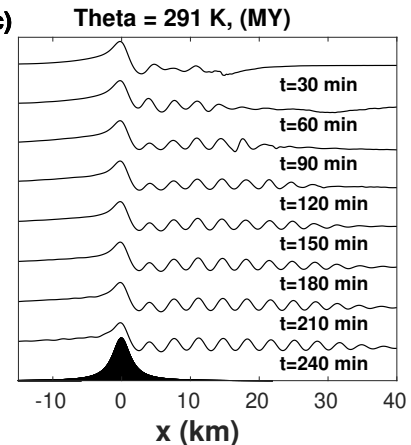

f) $R_{i}$ and theta with $\mathrm{J}=\mathbf{0 . 1 6}$, (MY)

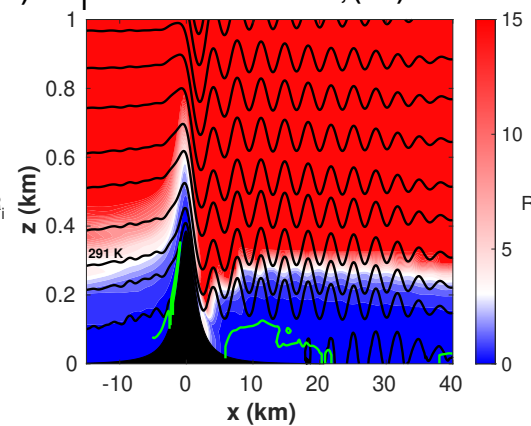

Figure 5: WRF simulation, for $J=0.16$ and $H_{N}=0.7$. Left to right : free slip, YSU BLYR and MY BLYR. Upper row : isotherm $\theta=291 \mathrm{~K}$ at different time. Lower row : large scale Richardson number (color), saturated at $R_{i f}=15$ for clarity. The blue color correspond to $R_{i f}<3$, green contours represent $R_{i f}<0.25$ and black contours are iso-lines of potential temperature 
a)

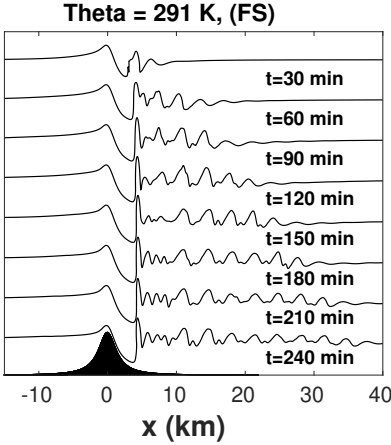

b)

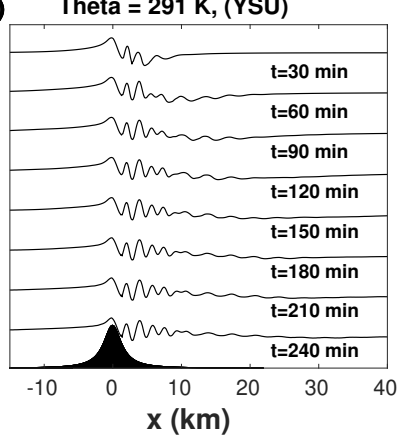

c) Theta $=291 \mathrm{~K},(\mathrm{MY})$

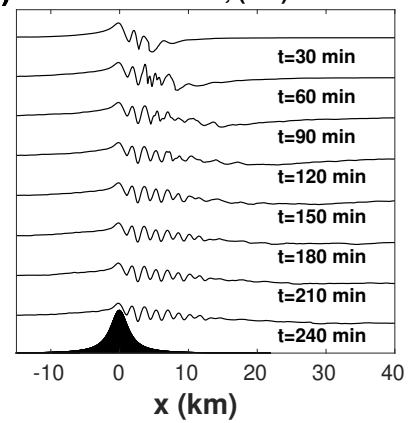

d) $\quad R_{i}$ and theta with $\mathrm{J}=5$, (FS)

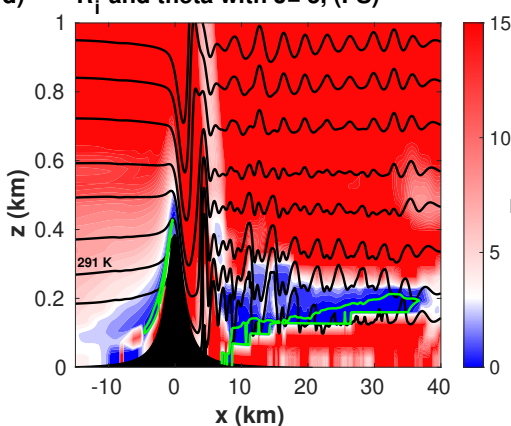

e) $\quad R_{\mathrm{i}}$ and theta with $\mathrm{J}=5$, (YSU)

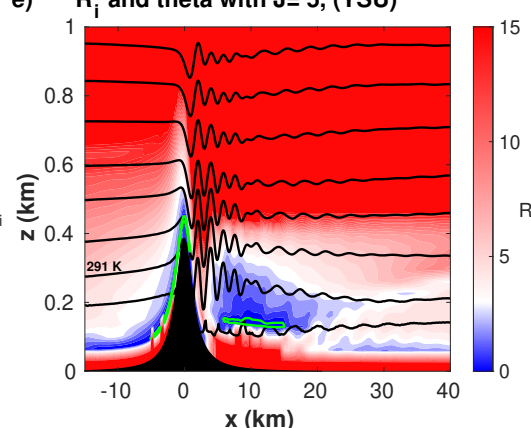

f) $\quad R_{i}$ and theta with $J=5$, (MY)

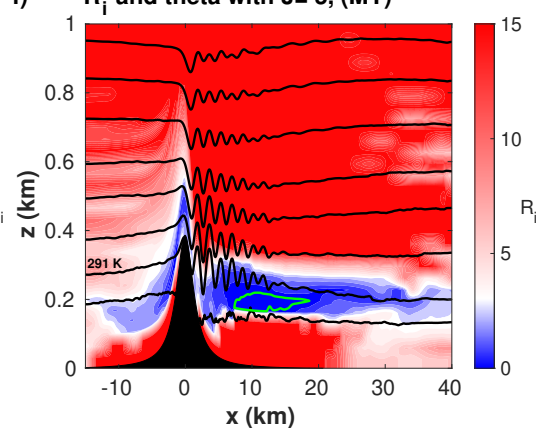

Figure 6: Same as Fig. 5 for $J=5$. 


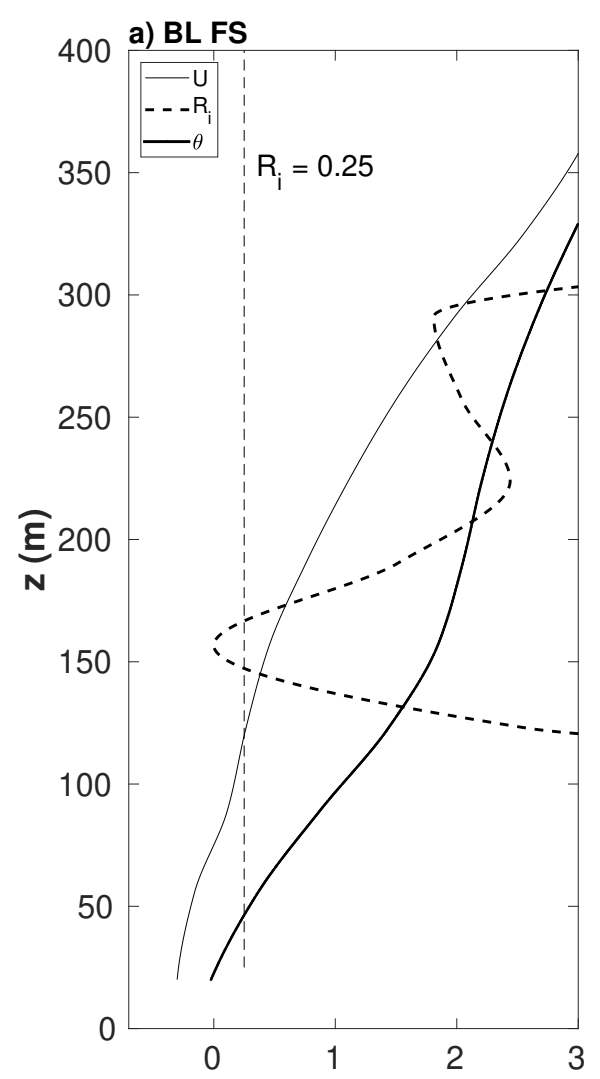

Theta-288 (K), U (m/s), Ri

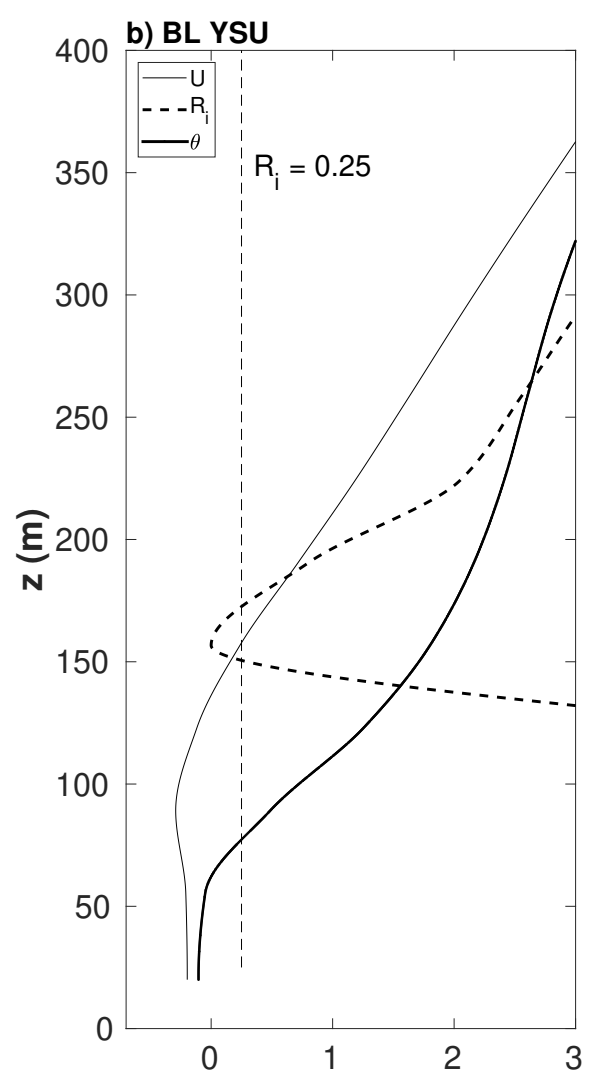

Theta-288 (K), U (m/s), Ri

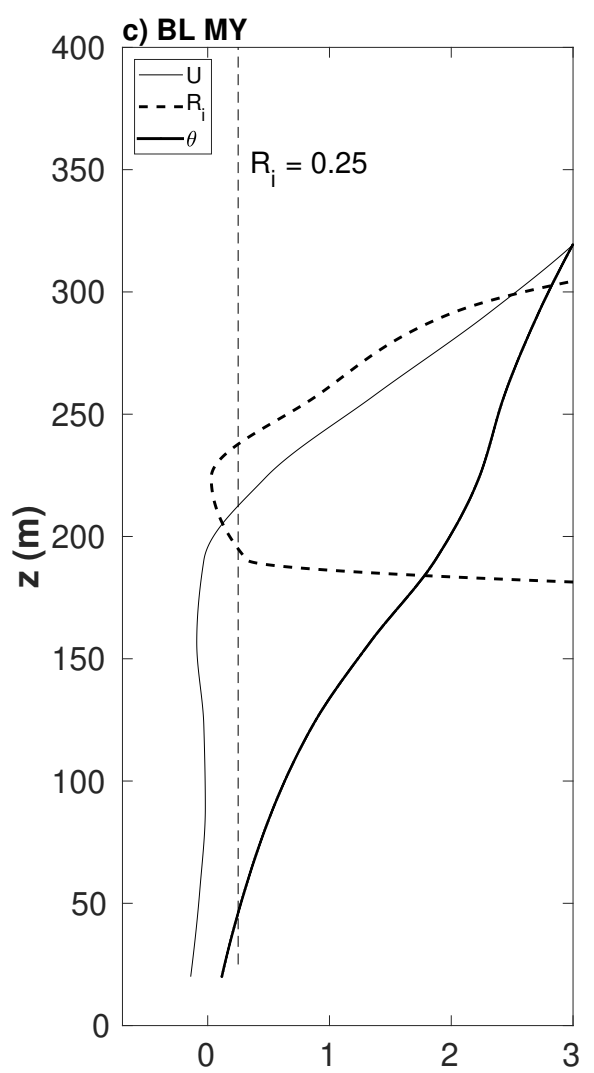

Theta-288 (K), U (m/s), Ri

Figure 7: Vertical profile of large scale horizontal velocity $U_{f}$, potential temperature $\theta_{f}-288$ $\mathrm{K}$ and Richardson number $R_{i f}, 13 \mathrm{~km}$ downstream the mountain. $H_{N}=0.7$ and $J=5$. The thin dashed line corresponds to $R_{i f}=0.25$ 